\title{
Improved management of systemic venous anomalies in a single ventricle: New rationale
}

\author{
Antonio Amodeo, MD, ${ }^{\mathrm{a}}$ Mauro Grigioni, $\mathrm{MS},{ }^{\mathrm{c}}$ Sergio Filippelli, MD, ${ }^{\mathrm{a}}$ Maria Giulia Gagliardi, MD, \\ Costantino Del Gaudio, PhD, ${ }^{\mathrm{d}}$ Umberto Morbiducci, $\mathrm{PhD},{ }^{\mathrm{e}}$ Giuseppe D’Avenio, MS, ${ }^{\mathrm{c}}$ \\ Gianluca Brancaccio, MD, and Roberto M. Di Donato, MD ${ }^{\mathrm{a}}$
}

\begin{abstract}
Objectives: Two innovative surgical approaches addressing systemic venous anomalies in single-ventricle patients are evaluated.

Methods: Between 2003 and 2007, 7 patients underwent a unifocal bilateral bidirectional cavopulmonary anastomosis, and 5 patients underwent a hepatoazygos venous connection associated with a previous $(n=4)$ or concomitant $(n=1)$ Kawashima operation. Computational fluid dynamics simulations allowed investigation of 2 sets of comparative models: (1) bifocal versus unifocal bilateral bidirectional cavopulmonary anastomosis and (2) classic hepatic vein-pulmonary artery channel versus hepatoazygos direct anastomosis for Fontan completion after or combined with the Kawashima operation.
\end{abstract}

Results: There was 1 hospital death in the unifocal bilateral bidirectional cavopulmonary anastomosis group. At a mean follow-up of $15.6 \pm 7.40$ months after a unifocal bilateral bidirectional cavopulmonary anastomosis and of $38.7 \pm 13.2$ months after direct hepatoazygos venous connection, respectively, all 11 survivors are in New York Heart Association class I with functional anastomoses. Computational assessment of bifocal bilateral bidirectional cavopulmonary anastomosis demonstrated weak perfusion between caval veins against symmetric and steady bilateral flow fields in the unifocal arrangement. In the classic post-Kawashima Fontan completion model, the hepatic venous flow to the pulmonary artery was held back by means of preponderant opposite flow, whereas in the direct hepatoazygos venous connection model, the hepatic venous flow merged smoothly into the azygos vein. Power-loss calculation showed no significant difference between bifocal and unifocal bilateral bidirectional cavopulmonary anastomosis topology, whereas the hepatoazygos connection clearly had better energy preservation than the classical connection.

Conclusions: This limited clinical and computational fluid dynamics assessment suggests the efficacy of this new rationale to reduce the additional thrombotic risks produced by systemic venous anomalies in single-ventricle patients.

Aberrant systemic venous connections that are mostly considered negligible in the management of 2-ventricle heart anomalies are of much greater surgical relevance when associated with single-ventricle cardiac malformations. In fact, conventional cavopulmonary anastomoses can be affected by the ensuing effects of competitive, unbalanced, or both systemic venous flows. Two conditions in particular, bilateral superior vena cava and interruption/azygos continuation

From the Cardiac Surgery Service ${ }^{\mathrm{a}}$ and the Cardiology Service ${ }^{\mathrm{b}}$ of the MedicalSurgical Department of Paediatric Cardiology, Bambino Gesù Paediatric Hospital, Rome, Italy; the Department of Technology and Health of the ISS (Italian National Institute of Health), ${ }^{\mathrm{c}}$ Rome, Italy; the Department of Chemical Science and Technology, ${ }^{\mathrm{d}}$ University of Rome “'Tor Vergata," Rome, Italy; and the Department of Mechanics, ${ }^{\mathrm{e}}$ Politecnico di Torino, Turin, Italy.

Read at the Eighty-seventh Annual Meeting of the American Association for Thoracic Surgery, Washington, DC, May 5-9, 2007.

Received for publication Feb 17, 2008; revisions received Dec 11, 2008; accepted for publication April 1, 2009; available ahead of print July 13, 2009

Address for reprints: Antonio Amodeo, MD, Dipartimento Medico Chirurgico di Cardiologia Pediatrica, Ospedale Pediatrico Bambino Gesù, Piazza S. Onofrio 4, 00165

Rome, Italy (E-mail: antonioamodeo@yahoo.it).

J Thorac Cardiovasc Surg 2009;138:1154-9

$0022-5223 / \$ 36.00$

Copyright $\odot 2009$ by The American Association for Thoracic Surgery

doi:10.1016/j.jtcvs.2009.04.039 of the inferior vena cava, can jeopardize the outcome of conventional cavopulmonary anastomoses. ${ }^{1-3}$

The presence of a bilateral superior vena cava might cause flow stagnation at the pulmonary artery confluence, with impaired growth of the central pulmonary arteries and increased risk of thrombus formation. ${ }^{1,2}$ In our earlier experience, a hypoplastic pulmonary artery confluence was seen in 2 of 11 patients who had received a bilateral bidirectional cavopulmonary anastomosis (BCPA). This entailed an extensive reconstruction of the pulmonary artery confluence at the time of the Fontan operation (unpublished data).

In the case of interruption and azygos continuation of the inferior vena cava undergoing Fontan completion after a Kawashima operation, either the intracardiac or extracardiac hepatic vein-pulmonary artery tubular connection is also potentially predisposed to stagnation and thrombosis. ${ }^{3}$ The latter is facilitated by an intrinsic hypercoagulable state ${ }^{4}$ and by the unbalanced ratio between the facing flows from the hepatic veins and the Kawashima cavopulmonary anastomosis, respectively. ${ }^{5}$ In our experience 4 of 8 such patients undergoing conventional hepatic vein-pulmonary artery 


\section{Abbreviations and Acronyms \\ BCPA = bidirectional cavopulmonary anastomosis \\ CFD = computational fluid dynamics \\ MRI = magnetic resonance imaging}

extracardiac conduit connection experienced failure of the Fontan principle for conduit thrombosis (unpublished data).

Recently, 2 innovative surgical approaches addressing these systemic venous " anomalies" in single-ventricle patients have been introduced at our institution: (1) the unifocal bilateral $\mathrm{BCPA}^{6}$ and (2) the hepatoazygos venous anastomosis, which was first reported by Baskett and colleagues ${ }^{7}$ and subsequently by others, including ourselves. ${ }^{5}$

This study comprises clinical information on a series of 12 patients undergoing operations with these new surgical solutions and parallel laboratory observations of their respective computational fluid dynamics (CFD) compared with conventional techniques.

\section{MATERIALS AND METHODS}

Between September 2003 and March 2008, 7 patients with a single ventricle and a bilateral superior vena cava underwent unifocal bilateral BCPA ( 1 in combination with a single-stage fenestrated Fontan operation); median age was 10.3 months (7.6-80.0 months), and median weight was $7.2 \mathrm{~kg}$ $(6.5-16 \mathrm{~kg})$. Six had pulmonary stenosis, and 2 had an intracardiac total anomalous pulmonary venous connection. Previous operations were placement of a systemic-pulmonary shunt $(\mathrm{n}=5)$ and pulmonary banding plus atrial septectomy $(\mathrm{n}=1)$.

Concurrently, another 5 patients received a hepatoazygos venous connection associated with a previous $(n=4)$ or concomitant $(n=1)$ Kawashima operation; median age was 38 months (12.8-118.6 months), and median weight was $10.2 \mathrm{~kg}(9-23 \mathrm{~kg})$. Cardiac diagnoses included left isomerism, single-ventricle and inferior vena caval interruption with azygos $(n=3)$ or hemiazygos $(n=2)$ continuation, pulmonary stenosis $(n=4)$, and dextrocardia $(n=3)$. Previous operations were placement of a systemic-pulmonary shunt $(\mathrm{n}=3)$ and pulmonary banding plus conal septal resection $(\mathrm{n}=1)$.

This study was approved by the scientific advisory board of the hospital relative to our ongoing studies on the best hydrodynamic patterns in the Fontan operation. ${ }^{8-10}$

\section{Surgical Technique}

The unifocal bilateral BCPA is a Y-shaped side-by-side anastomosis of the 2 transected superior venae cavae under the aortic arch followed by their end-to-side anastomosis, as a single vessel, to the pulmonary artery. ${ }^{6}$ Patch enlargement of the pulmonary arterial confluence was associated in 4 patients. The hepatoazygos venous connection is the direct anastomosis of the hepatic veins to the adjacent segment of the azygos/hemiazygos vein. ${ }^{5}$ Details of these surgical techniques are described elsewhere. ${ }^{5,6}$

\section{Follow-up}

Patients undergoing unifocal bilateral BCPA were dismissed on long-term antiaggregant therapy $\left(5 \mathrm{mg} \cdot \mathrm{kg}^{-1} \cdot \mathrm{d}^{-1}\right.$ aspirin), whereas patients with a hepatoazygos venous connection received anticoagulation for 6 months (dicumarol, aiming at an international normalized ratio of 2.0-3.0), followed by lifetime antiaggregant therapy. Midterm assessment included physical exam- ination, 2-dimensional/3-dimensional echocardiograms, cardiac catheterization, and magnetic resonance imaging (MRI) studies.

\section{CFD Studies}

CFD simulations were designed to investigate local hemodynamics and power dissipation in 2 sets of comparative models. The image-based computational models were obtained from the clinical data of the patients. Technically, MRI and angiographic data provided anatomic information on vessel geometries (eg, length, diameter, and out-of-plane curvature), as in previous in vitro studies on realistic glass-blown models of total cavopulmonary connection. ${ }^{8-10}$

Two geometric vascular connections were built simulating (1) classical bifocal bilateral BCPA and (2) unifocal bilateral BCPA, respectively. The distance between caval axes in configuration 1 was set at $40 \mathrm{~mm}$. Two other configurations were also generated: (3) classical hepatic vein-pulmonary artery channel and (4) hepatoazygos venous connection.

For all models, flow fields were computed by solving the 3-dimensional, steady, incompressible Navier-Stokes equations with the commercial CFD solver FLUENT (ANSYS, Inc, Lebanon, NH) with rigid vessel walls. ${ }^{8,10-14}$ Blood was assumed to be homogeneous and Newtonian (density of $1060 \mathrm{~kg} /$ $\mathrm{m}^{3}$ and dynamic viscosity of $3.5 \times 10^{-3} \mathrm{~Pa} \cdot \mathrm{s}$ ), and rigid walls were considered. Five-vessel-diameter-length flow extensions were added to minimize the effect of boundary conditions. As for the solution strategy, velocities were calculated according to the second-order upwinding scheme, and the pressure implicit splitting of operators scheme was adopted. The convergence criterion was set to $10^{-5}$ for all degrees of freedom.

Boundary conditions were also derived from hemodynamic and MRI data. For models 1 and 2, simulations were performed at 3 different flow rates specified as plug-flow velocity profiles at the 2 caval inflows (350, 700 , and $1000 \mathrm{~mL} / \mathrm{min}$ with 50:50 partition). The choice of a $350 \mathrm{~mL} /$ min flow condition represents the expected indexed caval flow of one patient of this series, whose angiographic findings were used for the CFD model. The other 2 incremental flow conditions were imposed to test the anastomosis at higher flow rates. A constant pressure of $8 \mathrm{~mm} \mathrm{Hg}$ was prescribed at the outlet section of both pulmonary arteries. ${ }^{8,9}$

Similarly for models 3 and 4, 3 different flow rates were prescribed at inflows $(1000,1500$, and $2000 \mathrm{~mL} / \mathrm{min}$ specified as plug-flow velocity profiles) and distributed as follows: in configuration 3 the hepatic and Kawashima anastomosis flows were $20 \%$ and $80 \%$ of the total venous return, respectively, and in configuration 4 the superior vena cava carried $30 \%$ of total venous return, and the remaining $70 \%$ resulted from the combined hepatic venous $(20 \%)$ and azygos venous $(50 \%)$ flows. Again, the choice of the cardiac outputs prescribed at inflows was based on the actual output of patients of the series (technically, $2000 \mathrm{~mL} / \mathrm{min}$ is the value of the patient who served as a model for the CFD study). In the case of models 3 and 4 , a stress-free condition was set at the pulmonary artery outflow, and thus the partition of the inlet flow rate between the right and the left pulmonary arteries was dictated only by the proximal impedances because of the geometry of the connection. In particular, the stress-free outflow boundary condition is given by the prescription of the following Neumann boundary condition at the outlet sections:

$$
p n-\mu \nabla V \cdot n=0,
$$

where $p$ is the pressure, $V$ is the velocity vector, $\mu$ is the dynamic viscosity, and $n$ is the outward normal to the outlet section.

The computational grids were generated by using GAMBIT (ANSYS, Inc): about 230,000 tetrahedral cells were used for both configurations of the bicaval connection, whereas 800,000 and 600,000 cells were chosen for the classic Kawashima and the hepatoazygos models, respectively. Sensitivity analysis was carried out to assess the proper grid refinement. Three different grids where used: difference in the averaged pressure decrease between inlets and outlets compared with the finest grid size model of less than $3 \%$ was considered satisfactory. 
Power dissipation across the connections was computed by using an integrated control volume balance:

$$
W_{\text {diss }}=W_{\text {in }}-W_{\text {out }}=\sum_{i=1}^{n_{I N}}\left(P_{i}+\frac{\rho}{2} U_{i}^{2}\right) Q_{i}-\sum_{j=1}^{n_{\text {OUT }}}\left(P_{j}+\frac{\rho}{2} U_{j}^{2}\right) Q_{i},
$$

where $W$ is power dissipation, $U$ is average velocity, $P$ is the static pressure, and $Q$ the volumetric flow rate in the considered vessel; the subscripts $I$ and $j$ refer to each input vessel and output vessel of the investigated anastomosis, respectively. ${ }^{9}$

\section{RESULTS}

\section{Clinical Results}

Among patients undergoing unifocal bilateral BCPA, there was 1 hospital death in the youngest patient because of a moderately obstructed intracardiac total anomalous pulmonary venous connection. Six patients had an uneventful postoperative course. Five were discharged with a median arterial oxygen saturation of $86 \%(80 \%-87 \%)$, whereas the patient who underwent a single-stage fenestrated Fontan operation and unifocal bilateral BCPA had a systemic arterial oxygen saturation of $93 \%$.

All patients receiving a hepatoazygos venous connection survived the operation and were fully oxygen saturated. Two required reoperation for bleeding, and 1 patient with congenital heart block had a stormy postoperative course and eventually recovered after a multisite pacing procedure.

At a mean follow-up of $15.6 \pm 7.40$ months (4-24 months) after unifocal bilateral BCPA and of $38.7 \pm 13.2$ months (25.1-60.0 months) after direct hepatoazygos venous connection, respectively, all patients are in New York Heart Association class I, with echocardiographic evidence of unobstructed and functional anastomoses.

Four patients with unifocal bilateral BCPA (Figure 1, A) and 4 with hepatoazygos venous connection (Figure 1,B) underwent postoperative cardiac catheterization, showing a mean cavopulmonary pressure of $11 \pm 2$ and $12 \pm 2$ $\mathrm{mm} \mathrm{Hg}$, respectively. All patients had good-sized central pulmonary arteries except 1 patient in the hepatoazygos venous connection group who required balloon pulmonary arterioplasty 4 months postoperatively. Three patients with hepatoazygos venous connections also received MRI evaluation, ruling out residual problems.

\section{CFD Results}

Bifocal bilateral BCPA versus unifocal bilateral BCPA models. Axial velocity vector plots of the 2 anastomoses in the transverse section are reported for the $350 \mathrm{~mL} / \mathrm{min}$ case. The bifocal configuration showed evident directionality of blood flow from either superior vena cava toward the ipsilateral pulmonary artery, with weak perfusion of the intercaval region (Figure 2, A). Conversely, in the unifocal configuration all portions of the central pulmonary arteries were perfused with symmetric and steady bilateral flow
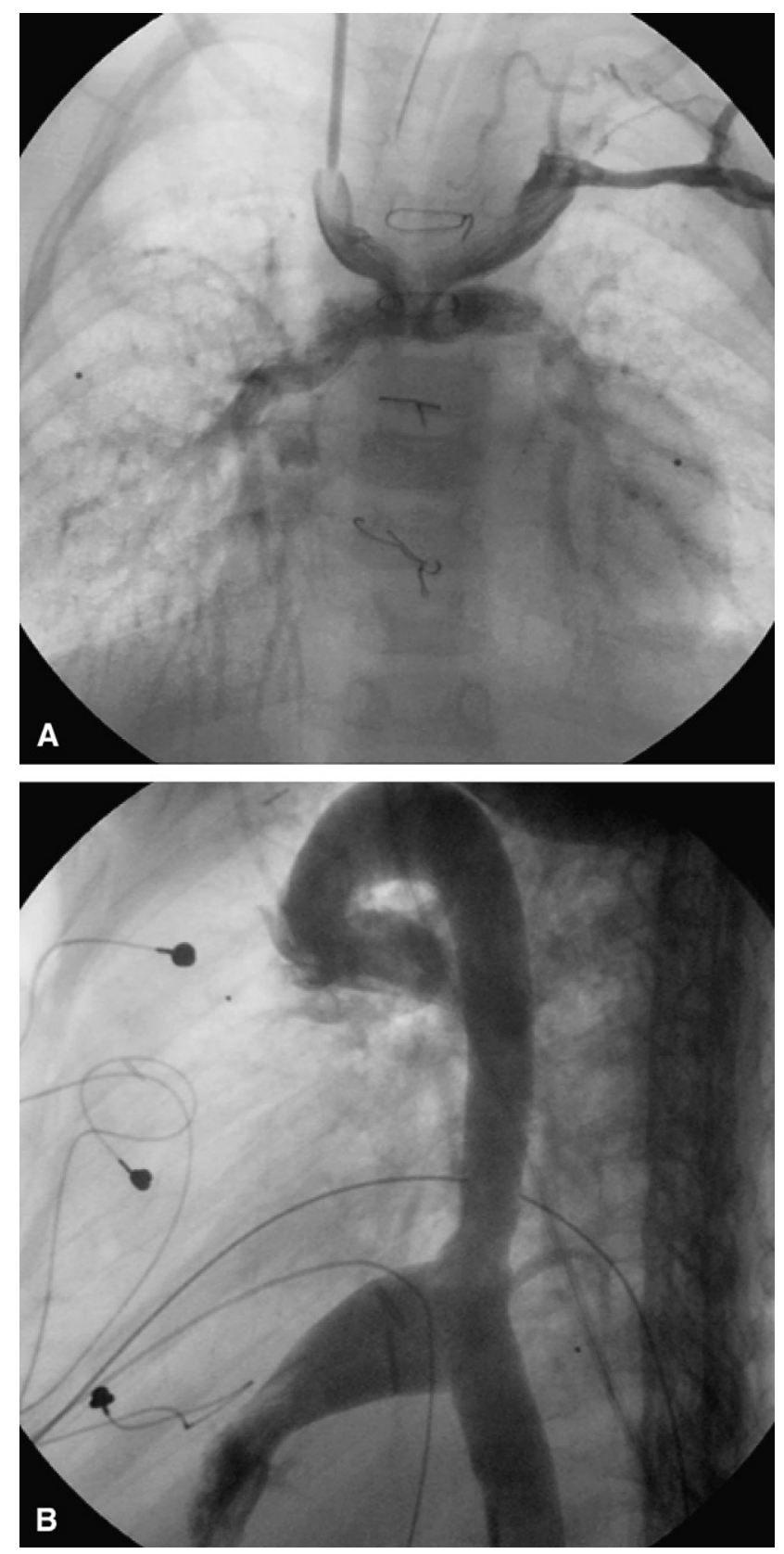

FIGURE 1. Angiographic appearance of unifocal bilateral bidirectional cavopulmonary anastomosis (A) and hepatoazygos venous connection (B).

fields (Figure 2, $B$ ). These findings were confirmed by means of 3-dimensional pathline visualizations (Figure 3). The unifocal bilateral BCPA configuration showed local blood mixing at the confluence of the caval veins into the pulmonary arteries and the absence of large recirculation zones in the whole anastomosis. Estimated pressure decreases across the computational models between caval veins and pulmonary arteries were less than $1 \mathrm{~mm} \mathrm{Hg}$ for all fluid dynamic conditions investigated, being consistent with clinical measurements. 

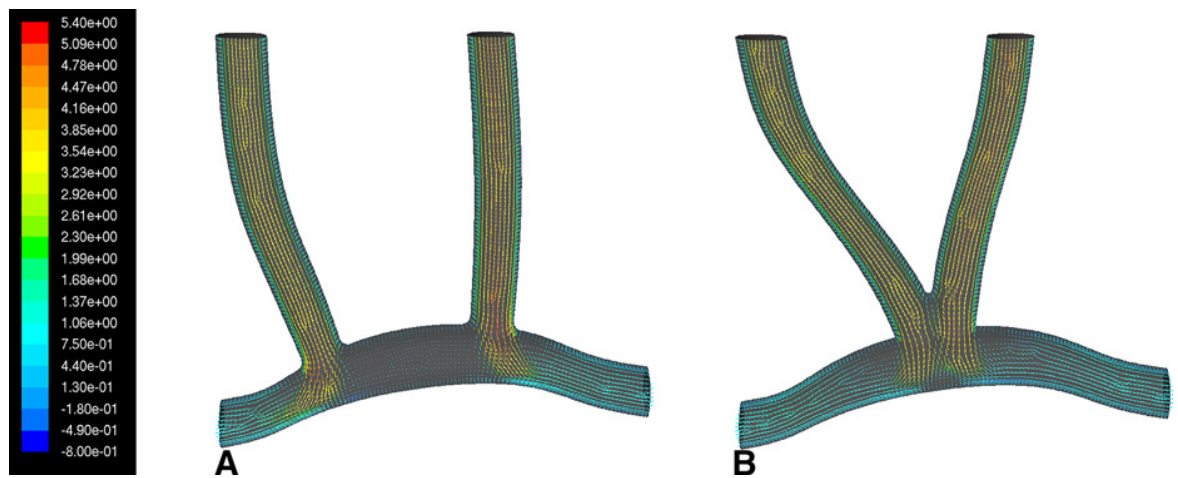

FIGURE 2. Axial velocity vector plots in the transverse section of the 2 investigated configurations with bilateral superior vena cava: A, bifocal bilateral bidirectional cavopulmonary anastomosis; B, unifocal bilateral bidirectional cavopulmonary anastomosis. The velocity scale is defined as centimeters per second.

Hepatoazygos versus hepatopulmonary connection models. The calculated flow field in the direct hepatoazygos venous connection model shows that the hepatic venous flow merges smoothly into the azygos vein (Figure 4, A). Further downstream, at the superior vena cava-pulmonary artery anastomosis level, the flow splits orderly and evenly in both directions (Figure 4, A). On the contrary, in the classic hepatic vein-pulmonary artery channel model, a relatively low flow moves upward in a rather large conduit, becoming predisposed to form an unorganized structure, particularly in the proximity of the cross (Figure $4, B$ ). This unfavorable flow pattern is further worsened by collision with the preponderant BCPA venous return.

Hydrodynamic power loss. In the case of a bilateral superior vena cava, power-loss calculation, using the same control volume, showed no appreciable difference between bifocal and unifocal topology. In fact, differences between the 2 BCPA topologies were negligible, as observed in the entire range of flows considered $(350,700$, and $1000 \mathrm{~mL} /$ min; Figure 5, A).

In the case of azygos continuation, the hepatoazygos venous connection showed a clear advantage over the tradi- tional alternative (Figure 5, B). A relevant role was played by the distensibility of the azygos vein and by the amount of additional flow. In fact, the diameter of the azygos vein increased after the anastomosis of the hepatic veins. According to the Poiseuille formula, vessel diameter greatly influences the pressure in a tube. Therefore the increased diameter of the azygos vein remarkably decreases the energy losses.

\section{DISCUSSION}

Peculiar systemic venous connections are known to jeopardize the outcome of conventional cavopulmonary anastomoses through essentially 2 mechanisms: (1) flow disturbance and (2) flow imbalance.

The presence of a bilateral Glenn anastomosis produces stagnation in the pulmonary arterial confluence. Consequently, it might impair the growth of the central pulmonary arteries and even promote thrombus formation at their level, affecting the eligibility of these patients for Fontan completion. ${ }^{1,2}$ Anecdotal reports have advocated technical expedients to avoid bilateral BCPA. Although ingenious, these techniques might occasionally lack feasibility (eg, in the
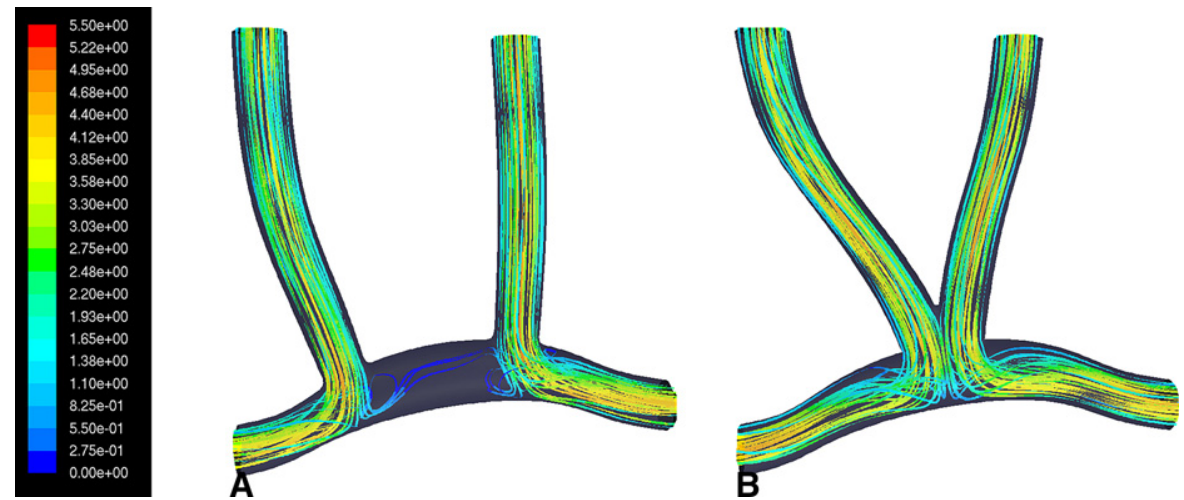

FIGURE 3. Pathline visualization: A, bifocal bilateral bidirectional cavopulmonary anastomosis; B, unifocal bilateral bidirectional cavopulmonary anastomosis. The pathlines are color coded according to the velocity magnitude (in centimeters per second). 

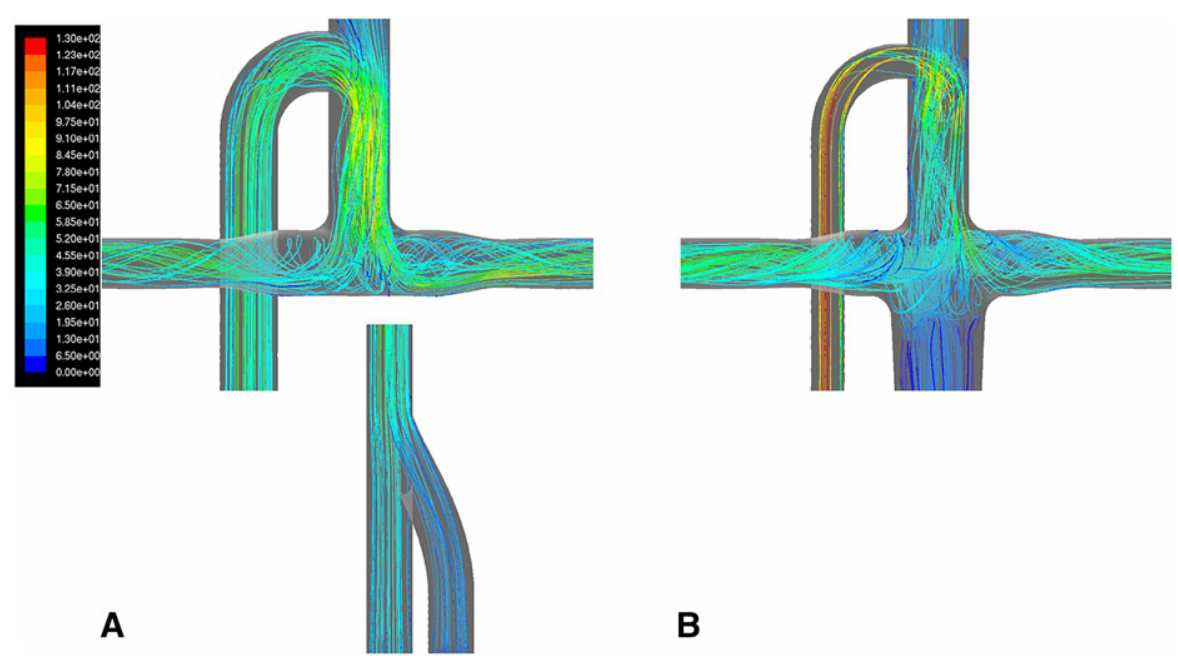

B

FIGURE 4. Pathline visualization: A, Kawashima cavopulmonary anastomosis after incorporation of the hepatoazygos venous connection; B, Kawashima cavopulmonary anastomosis with the classical hepatic vein-pulmonary artery conduit connection. The pathlines are color coded according to the velocity magnitude (in centimeters per second).

case of creation of an end-to-side cavocaval connection) or reliability (eg, in the case of early obliteration of the lesser superior vena cava or of interposition of a prosthetic innominate vein). ${ }^{15}$

Patients with left isomerism, a single ventricle, and azygos continuation of the inferior vena cava undergoing a conventional Fontan completion by means of conduit interposition between the hepatic veins and the pulmonary circulation are even more susceptible to thrombosis. ${ }^{3}$ Pathogenesis of this thrombogenic tendency is 2-fold. On the one hand, these patients carry a genetic hypercoagulable condition, as confirmed by an intrinsic tendency for deep vein thrombosis in the presence of an inferior vena caval interruption. ${ }^{4}$ On the other hand, a relatively low upward flow from the hepatic veins $(\leq 20 \%$ of total systemic venous return) is literally held back by the preponderant downward flow of the Kawashima BCPA (ie, flow unbalance mechanism).

This study endorses the application of 2 innovative surgical techniques for the management of systemic venous aber- rancies in combination with cavopulmonary anastomoses. Two principles underlie these techniques.

First, the venous unifocalization principle, achieved by means of creating a side-by-side anastomosis between the transected bilateral superior venae cavae or by means of creating an end-to-side anastomosis between hepatic veins and the nearby segment of the azygos vein, respectively, essentially aims at reproducing the normal anatomic pattern of the systemic venous system in which all tributaries converge, at a more or less acute angle, into vessels of progressively larger caliber, ending with a single terminal caval vessel.

Second, the cavopulmonary polarization principle pursues the best spatial arrangement of opposite systemic venous connections with the pulmonary arteries in an attempt to avoid fluid collision and minimize power dissipation. ${ }^{10}$ The rationale for unifocal bilateral BCPA is that a single and centrally located cavopulmonary connection provides an unopposed and symmetric bilateral distribution of pulmonary blood flow, thus yielding growth of the central
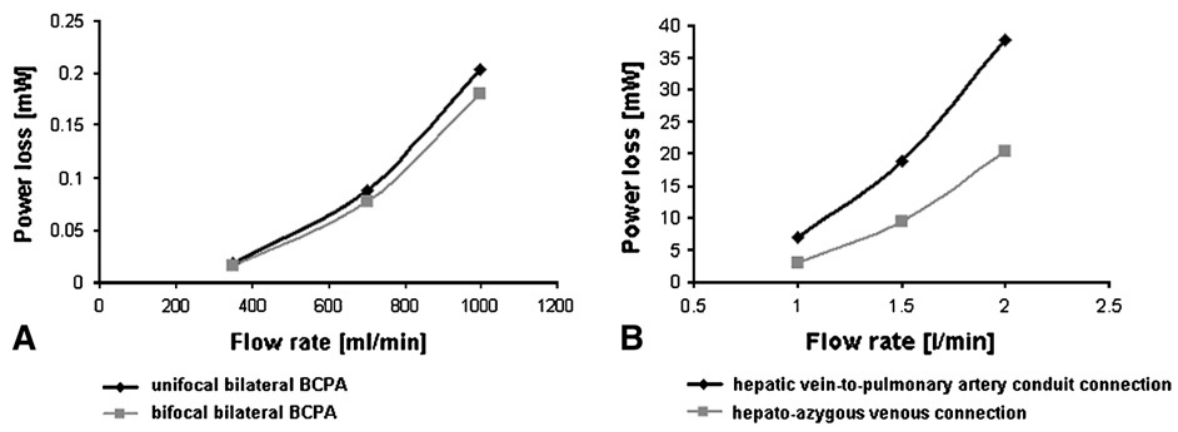

FIGURE 5. Power-loss versus flow rate plots for the anatomic models investigated: A, Kawashima cavopulmonary anastomosis after incorporation of the hepatoazygos venous connection; B, Kawashima cavopulmonary anastomosis with the classical hepatic vein-pulmonary artery conduit connection. $B C P A$, Bidirectional cavopulmonary anastomosis. 
pulmonary arteries and prevention of thrombus formation. ${ }^{4}$ In the case of direct hepatoazygos connection, the "unipolarization" of the systemic venous return (the single cavopulmonary anastomosis) effectively obviates the described problem of unbalanced cavopulmonary connections by forcing the entire systemic venous return into a single outlet. ${ }^{5}$

Except for a single death that was unrelated to technical issues, the results of this small series prove the feasibility, safety, and efficacy of the 2 new procedures. Admittedly, from a surgical point of view, both techniques present pitfalls and require a good deal of geometric envisioning. ${ }^{5,6}$ For example, considering that bilateral superior venae cavae are intuitively smaller than a single superior vena cava, it is preferable to perform the unifocal bilateral BCPA in children weighing $6 \mathrm{~kg}$ or greater, a patient size that ensures a relatively good caliber of the 2 vessels and a little more extra tissue for their central merging. In addition, the hepatoazygos venous connection is more easily accomplished in combination with the Kawashima anastomosis, preferably at an age of 1 year or greater, avoiding the nuisance of sternal reentry.

The midterm clinical outcome of either technique is encouraging, and both imaging techniques and hemodynamic assessment show proper function of the anastomoses.

The unifocal bilateral BCPA was not found to ensure better energy preservation than its conventional counterpart. On the other hand, the hepatoazygos connection showed a clear advantage over the classical Kawashima connection in energetic terms.

Nevertheless, both axial velocity vector plots and pathline distributions show no relevant flow disturbance in either unifocal bilateral BCPA or hepatoazygos venous connection (reduced low-velocity regions or chaotic mixing, respectively). Ultimately, this translates into avoiding flow stagnation in either the central pulmonary arteries, which is typical of classical bifocal bilateral BCPA, or in the hepatic veinpulmonary artery conduit in the case of conventional Fontan completion after the Kawashima operation.

\section{CONCLUSION}

This limited clinical information and the CFD studies suggest that both unifocal bilateral BCPA and hepatoazygos venous connection are safe and effective surgical techniques for the treatment of systemic venous anomalies in patients with a single ventricle. They both improve flow patterns in the cavopulmonary system and presumably reduce the risk of thrombosis. However, further clinical and flow studies will be necessary to demonstrate a true physiologic advantage of either technique.

\section{References}

1. McElhinney DB, Reddy VM, Moore P, Hanley FL. Bidirectional cavopulmonary shunt in patients with anomalies of systemic and pulmonary venous drainage. Ann Thorac Surg. 1997;63:1676-84.

2. Ganeshakrishnan K, Iyer GKT, Van Arsdell GS, Dicke FP, McCrindle BW, Coles JG, et al. Are bilateral superior vena cavae a risk factor for single ventricle palliation? Ann Thorac Surg. 2000;70:711-6.

3. Konstantinov IE, Puga FJ, Alexi-Meskishvili VV. Thrombosis of intracardiac or extracardiac conduits after modified Fontan operation in patients with azygous continuation of the inferior vena cava. Ann Thorac Surg. 2001;72:1641-4.

4. Mouton WG, Zehnder T. Deep vein thrombosis in azygos continuation. Eur J Vasc Endovasc Surg. 2003;25:90-2.

5. Amodeo A, Di Carlo D, Grigioni M, De Santis M, Di Donato R. Early primary Kawashima operation combined with direct hepatic vein-to-azygos vein connection: a new logical approach. J Thorac Cardiovasc Surg. 2005;129:949-50.

6. Amodeo A, Di Donato RM. The confluent bilateral bidirectional anastomosis. The unifocal bilateral bidirectional cavopulmonary anastomosis. Ann Thorac Surg. 2007;84:2134-5.

7. Baskett RJF, Ross DB, Warren AE, Sharrat GP, Murphy DA. Hepatic vein to the azygous vein anastomosis for pulmonary arteriovenous fistulae. Ann thorac Surg. 1999;68:232-3.

8. Grigioni M, Daniele C, Del Gaudio C, Morbiducci U, Balducci A, D'avenio G, et al. Numerical simulation of a realistic total cavo-pulmonary connection: effect of unbalanced pulmonary resistances on hydrodynamic performance. Int $J$ Artif Organs. 2003;26:1005-14.

9. Grigioni M, D'Avenio G, Amodeo A, Di Donato RM. Power dissipation associated with surgical operations' hemodynamics: critical issues and application to the total cavopulmonary connection. J Biomech. 2006;39:1583-94.

10. Amodeo A, Grigioni M, Oppido g Daniele C, D'Avenio G, Pedrizzetti G, et al. The beneficial vortex and best spatial arrangement in total extracardiac cavopulmonary connection. J Thorac Cardiovasc Surg. 2002;124:471-8.

11. Pekkan K, de Zélicourt D, Ge L, Sotiropoulos F, Frakes D, Fogel MA, et al. Physics-driven CFD modeling of complex anatomical cardiovascular flows-a TCPC case study. Ann Biomed Eng. 2005;33:284-300.

12. Dubini G, de Leval MR, Pietrabissa R, Montevecchi FM, Fumero R. A numerical fluid mechanical study of repaired congenital heart defects. Application to the total cavopulmonary connection. J Biomech. 1996;29:111-21.

13. de Zélicourt DA, Pekkan K, Parks J, Kanter K, Fogel M, Yoganathan AP. Flow study of an extracardiac connection with persistent left superior vena cava. J Thorac Cardiovasc Surg. 2006;131:785-91.

14. de Leval MR, Dubini G, Migliavacca F, Jalali H, Camporini G, Redington A, et al. Use of computational fluid dynamics in the design of surgical procedures: application to the study of competitive flows in cavopulmonary connections. J Thorac Cardiovasc Surg. 1996;111:502-13.

15. Vida VL, Leon-Wyss J, Garcia F, Castañeda AR. A Gore-Tex “'new-innominate' vein: a surgical option for complicated bilateral cavopulmonary shunts. Eur J Cardiothorac Surg. 2006;29:112-3. 